\title{
A Combined Approach to the Treatment of Adults with Osteogenesis Imperfecta
}

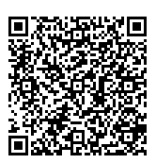

\author{
Jay R Shapiro*1 and Paul D Sponseller ${ }^{2}$ \\ ${ }^{1}$ Department of Medicine, Uniformed Services University of the Health Sciences, USA \\ ${ }^{2}$ Division of Pediatric Orthopaedic Surgery, Johns Hopkins Medical Institutions, USA
}

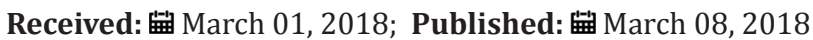

*Corresponding author: Jay R. Shapiro, Department of Medicine, Uniformed Services University of the Health Sciences, Bethesda, USA.

\section{Mini Review}

Osteogenesis Imperfecta (OI) is an inherited disorder of bone characterized by bone fragility and increased fracture risk affecting approximately 25,000 children and adults in the US [1]. OI is caused by different gene mutations involving the synthesis of type I collagen alpha chains. Recently, mutations affecting post translational processing of type I collagen as well as several non-collagenous proteins (SP7. Osterix) involved in the Wnt signalling pathway have been recognized. To date, 19 genes are implicated in osteogenesis imperfecta phenotypes [2]. In individuals with OI fractures occur throughout the lifetime, more frequently at the extremes of age. Fractures in OI may first be recognized in ultrasound studies during pregnancy but fracture risk while increased in childhood, tends to decrease following puberty only to increase around age 50 in both women and men. Compared to adult osteoporosis, there have been no systematic studies evaluating optimal daily intake of calcium or vitamin D on BMD or fracture incidence in adults. Current use is highly variable in adults who: a) either do not regularly take supplements or, b) take calcium and vitamin D without, measurement of serum 25(OH)D levels or urinary calcium excretion. Both hypercalcuria and renal stones occur in adults with OI although the incidence is not reported. Furthermore there is little documentation of vitamin D levels in adults with OI.

An extensive literature describes effective pharmacologic treatment of OI in children. Initially based on treatment with a second generation bisphosphonate pamidronate, treatment options have expanded to include third generation bisphosphonate zoledronic acid as well as denusomab, a RANK ligand inhibitor [3]. Teriparatide (hrPTH) use is restricted in children.

However, the indications for pharmacological treatment in adults with OI is less well defined than in children. Symptoms directing the physician towards initiating treatment include ongoing fracture rate, generalized bone pain and limitation in mobility affecting activities of daily living.
For that reason, physicians tend to rely on current adult osteoporosis treatment guidelines [4]. Treatment regimens include bisphosphonates used orally (alendronate, residronate) or by parenteral administration (pamidronate, denusomab and teriparatide). As an example, a recent study comparing teriparatide vs.intravenious neridronate, a third generation bisphosphonate, reported that while BMD changes at the lumbar spine at 24 months were $5.1 \%$ with teriparatide vs $-1.6 \%$ with neridronate $(\mathrm{P}<0.001)$ there was no significant difference in new fractures during the 24-month treatment period [4]. Note; There was no control group in this study [5]. Treatment with anti-sclerostin antibody, recently shown effective in improving bone density and decreasing fracture rate in adult osteoporosis, is the subject of a current phase 3 treatment protocol (Mereo Pharmaceutical).

Fractures are the hallmark of OI and thus, orthopaedic care is the mainstay of treatment: Coordination of orthopaedic and medical care is important in order to limit fractures and the time constrained by disability. It is useful for the orthopaedic surgeon to know about prior treatment with drugs such as bisphosphonates or teriparatide when surgical repair of a fracture is required as prolonged treatment with these drugs will alter bone quality and may affect repair. In OI the incidence of lifetime surgical intervention in adults approximates an estimated 60-70\%. Surgical repair of fractures or long bone deformities in adults with OI may present various difficulties. Low bone mineral density, skeletal or joint deformities, or instrumentation from previous surgeries complicates surgical stabilization of fractures. Furthermore, the adult with OI may present with post-traumatic or accelerated degenerative joint disease, kyphoscoliosis, and spondylolisthesis. Pre and post operative anaesthesia care, including the process of intubation, is a major problem particularly in patients with severe, restrictive pulmonary disease or tracheal deformity. 
Orthopedic surgery in OI may have 4 major roles: to treat fracture; to prevent recurrent fractures by adding long stabilizing implants; to correct deformity of long bones or spine; and to treat degenerative joint disease [4]. In all these cases, internal fixation by long intramedullary implants is preferred since it minimizes stress changes in the bone. Continued improvements in implants have decreased the risk of re-operation. Spine surgery is indicated for severe scoliosis or kyphosis to prevent pulmonary restriction or slippage of the lumbosacral joint. Fixation with multiple pedicle screws is an advance over the past decade which provides better correction and stability than wires or hooks [6]. Basilar invagination at the craniocervical junction is a silent but progressive threat in a minority or patients which may cause spasticity and weakness. In severe cases surgical decompression and stabilization is indicated.

Arthritis of large joints is more common in OI than in the general population, and occurs at an earlier age. It most commonly affects the hips and the knees. Protrusion of the femoral head in the acetabulum may accompany hip disorders. Although conservative care is the mainstay of treatment, total joint arthroplasty has been successful in many patients with type 1 OI. Over time, orthopedic/ medical issues tend to pose an increasing problem for the adult with OI. Skeletal deformities impair daily activities pulmonary function tends to decline with age and the risk of fracture increases with age. It important that the patient can rely on an accessible, knowledgeable, interactive and affordable program of health care.

\section{References}

1. Shapiro, J R, Byers P, Glorieux F, Sponseller P (2015) Osteogenesis imperfecta: a translational approach to brittle bone disease Elsevier/ Academic Press, San Diego, USA.

2. Lim J, Grafe I, Alexander S, Lee B (2017) Genetic causes and mechanisms of Osteogenesis Imperfecta. Bone 102: 40-49.

3. Palomo T, Vilaça T, Lazaretti-Castro M (2017) Osteogenesis imperfecta: diagnosis and treatment. Curr Opin Endocrinol Diabetes Obes. 24(6): 381-388.

4. Qaseem A, Forciea MA, McLean RM, Denberg TD, Clinical Guidelines Committee of the American College of Physicians. (2017) Treatment of low bone density or osteoporosis to prevent fractures in men and women: a clinical practice guideline update from the American College of Physicians. Ann Intern Med. 166(11): 818-839.

5. Leali PT, Balsano M, Maestretti G, Brusoni M, Amorese V, et al. (2017) Efficacy of teriparatide vs neridronate in adults with osteogenesis imperfecta type I: a prospective randomized international clinical study. Clin Cases Miner Bone Metab. 14(2):153-156.

6. Yilmaz G, Hwang S, Oto M, Kruse R, Rogers KJ, et al. (2014) Surgical treatment of scoliosis in osteogenesis imperfecta with cementaugmented pedicle screw instrumentation. J Spinal Disord Tech 27(3): 174-80.

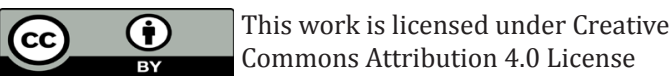

To Submit Your Article Click Here: Submit Article

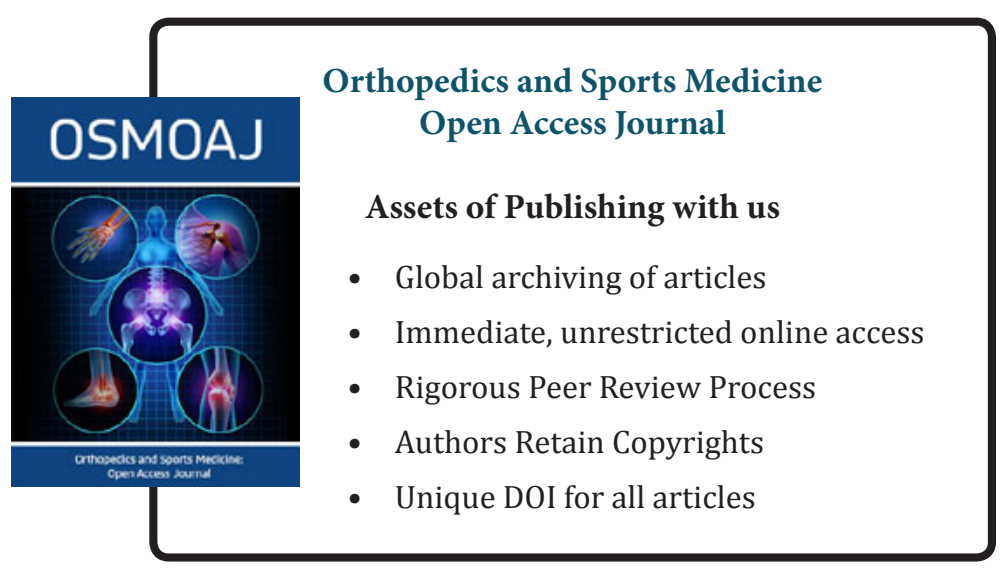

